SUSX-TH/02-022

hep-th/0210026

\title{
Moving Five-Branes and Cosmology *
}

\author{
André Lukas \\ Centre for Theoretical Physics \\ University of Sussex \\ Falmer, Brighton BN1 9QJ, United Kingdom \\ E-mail: a.lukas@sussex.ac.uk
}

\begin{abstract}
We discuss low-energy heterotic M-theory with five-branes in four and five dimensions and its application to moving brane cosmology.
\end{abstract}

\section{Introduction}

If string/M-theory is the correct fundamental theory of nature time-evolution of branes is likely to have played an important role in the history of the early universe. In this talk, I will review several aspects of this early universe brane dynamics in the context of strongly-coupled heterotic string-theory. This theory can be formulated as 11-dimensional supergravity on the orbifold $S^{1} / Z_{2}$ coupled to two 10-dimensional $E_{8}$ super-Yang-Mills multiplets localised on the two orbifold fixed planes (or boundaries) [1]. Upon compactification on a CalabiYau three-fold one arrives at a five-dimensional $N=1$ supergravity theory on $S^{1} / Z_{2}$ where the now four-dimensional boundaries carry $N=1$ gauge-theories which originate from the 10-dimensional Yang-Mills theories [2, 3]. Further, M-theory five-branes can be included in this compactification. They wrap twocycles within the internal Calabi-Yau space and, hence, appear as three-branes from the viewpoint of the effective five-dimensional theory. It is the dynamics of these $\mathrm{M}$ five-branes/three-branes which we are going to focus on in the following.

${ }^{*}$ Based on a talk given at the 1st International Conference on String Phenomenology, Oxford, 6-11 Jul 2002. 
It should be stressed that our approach to early universe cosmology has the virtue of being embedded in a meaningful and successful environment for particle phenomenology from M-theory. Specifically, one can construct models within the class of compactifications described above which lead to phenomenologically promising theories close to the standard model of particle physics located on one of the boundaries $[4,5]$.

The plan of this talk is as follows. To set the scene, I will present the effective five- and four-dimensional theories of heterotic M-theory including threebranes in their minimal versions. The status and interpretation of these effective theories as well as their general implications for three-brane dynamics will be reviewed. Subsequently, specific classes of solutions, both in four and five dimensions, describing simple three-brane evolution will be presented. Finally, we will discuss a new approach to brane-dynamics where three-branes are modelled as topological defects (kinks) of a bulk scalar field theory. Within this approach, more complicated brane evolution can be analysed by studying the dynamics of the modelling bulk scalar field. Particularly, what amounts to topologychanging transitions of the original M-theory action can be explicitly described by certain transitions within this bulk scalar field theory.

\section{Effective actions in five and four dimensions}

The five-dimensional effective action for heterotic M-theory is given by a specific $D=5, N=1$ gauged supergravity in the bulk coupled to four-dimensional $N=1$ gauge theories on the boundaries and the three-branes. In its minimal version there are two bulk supermultiplets, namely the supergravity multiplet containing the metric, the gravi-photon and the gravitini and the universal hypermultiplet containing the dilaton $\Phi$ along with three pseudo-scalar partners and the associated fermions. On the boundaries $M_{4}^{i}$, where $i=1,2$, one obtains $N=1$ gauge theories with gauge groups $G_{i} \subset E_{8}$ and chiral matter fields transforming under $G_{i}$. The details of this boundary field content depend on the compactification from eleven to five dimensions which is the main subject of particle physics model building in heterotic M-theory. Each three-brane $M_{4}^{3}$ (and its $Z_{2}$ mirror $\tilde{M}_{4}^{3}$ ) carries on its world-volume a universal chiral multiplet containing the field $Y$, specifying the three-brane position in the transverse space, along with an axionic partner and $U(1)$ vector multiplets. The number of these vector multiplets is given by the genus $g$ of the curve wrapped by the M five-brane.

The effective action for these fields $[2,3]$ can be consistently truncated to an even simpler version containing the metric $g_{\alpha \beta}$ and the dilaton $\Phi$ as the only

bulk fields and the embedding coordinates $X^{\alpha}=X^{\alpha}\left(\sigma^{\mu}\right)$ as the only brane fields. For a single three-brane, the associated effective action is then given by

$$
S_{5}=-\frac{1}{2 \kappa_{5}^{2}}\left\{\int_{M_{5}} \sqrt{-g}\left[\frac{1}{2} R+\frac{1}{4} \partial_{\alpha} \Phi \partial^{\alpha} \Phi+\frac{1}{3} \alpha^{2} e^{-2 \Phi}\right]\right.
$$




$$
\begin{aligned}
& +\int_{M_{4}^{1}} \sqrt{-g} 2 \alpha_{1} e^{-\Phi}+\int_{M_{4}^{2}} \sqrt{-g} 2 \alpha_{2} e^{-\Phi} \\
& \left.+\int_{M_{4}^{3} \cup \tilde{M}_{4}^{3}} \sqrt{-\gamma} \alpha_{3} e^{-\Phi}\right\} .
\end{aligned}
$$

where $\alpha_{i}=\sigma \beta_{i}, \beta_{i} \in \mathbf{Z}$ for $i=1,2,3$ are the charges on the boundaries and the three-brane quantised in units of $\sigma$. These charges satisfy the cohomology condition

$$
\sum_{i=1}^{3} \alpha_{i}=0
$$

The gauge-charge $\alpha$ is defined as a sum of step-functions

$$
\alpha=\alpha_{1} \theta\left(M_{4}^{1}\right)+\alpha_{2} \theta\left(M_{4}^{2}\right)+\alpha_{3}\left(\theta\left(M_{4}^{3}\right)+\theta\left(\tilde{M}_{4}^{3}\right)\right) .
$$

Note that two actions of the above type with two different sets of charges $\alpha_{i}$ correspond to topologically different M-theory compactifications.

The four-dimensional effective action obtained from (1) by compactifying on the orbifold is given by

$$
S_{4}=-\frac{1}{2 \kappa_{P}^{2}} \int_{M_{4}} \sqrt{-g_{4}}\left[\frac{1}{2} R_{4}+\frac{1}{4} \partial_{\mu} \phi \partial^{\mu} \phi+\frac{3}{4} \partial_{\mu} \beta \partial^{\mu} \beta+\frac{q_{3}}{2} e^{\beta-\phi} \partial_{\mu} z \partial^{\mu} z\right]
$$

where $q_{3}=\pi \rho \alpha_{3}$. Here, the field $\phi$, as the zero mode of the five-dimensional dilaton $\Phi$ measures the Calabi-Yau size, while the field $\beta$ originates from the (55) component of the metric and measures the orbifold size. Finally, the field $z$ specifies the three-brane position and is normalised so that $z \in[0,1]$ with $z=0,1$ corresponding to the two boundaries. This effective action can be obtained from the Kähler potential [6]

$$
K=-\ln \left(S+\bar{S}-q_{3} \frac{(Z+\bar{Z})^{2}}{T+\bar{T}}\right)-3 \ln (T+\bar{T}),
$$

by truncating off the axionic fields contained in the chiral superfields $S \leftrightarrow \phi$, $T \leftrightarrow \beta$ and $Z \leftrightarrow z$. Perturbatively, these moduli superfields are flat directions but one expects non-perturbative contributions to their superpotential from a number of sources $[7,8]$. Assuming the axions can still be integrated out, these contributions can be included by adding a suitable potential $V=V(\phi, \beta, z)$ to the above action. Note that this potential will be a function of all three fields rather than $z$ alone. It is quite clear even from the free action (4) that three-brane evolution in a static geometry (that is, for constant $\phi$ and $\beta$ ) is not possible. For this reason, the above action (possibly with a potential) represents the minimal system in which heterotic moving-brane cosmology can be discussed in a meaningful way. 


\section{Cosmological solutions with a moving three- brane}

The most general cosmological solutions of (4) with flat spatial sections [9] show a number of interesting generic features. Asymptotically at early and late time, both in the negative and positive time branch, the three-brane is practically at rest while the dilaton $\phi$ and the T-modulus $\beta$ evolve according to a standard rolling radii solution. However, the early and late rolling radii solutions are generally different. Interpolation between these rolling radii solutions is achieved at intermediate time due to non-trivial evolution of the three-brane. It turns out that not all rolling radii solutions can be approached asymptotically but only those for which the strong-coupling expansion parameter

$$
\epsilon \sim e^{\beta-\phi}
$$

diverges at early and late time. The brane-evolution, therefore, drives the system to strong coupling asymptotically. This is a direct consequence of the nontrivial kinetic term for $z$ in the action (4) and it is a feature that one would have missed had one - inconsistently - studied the brane-evolution in a static geometry.

Further, for all solutions the three-brane moves for a finite coordinate distance only. As a consequence, the brane may or may not collide with one of the boundaries, depending on initial conditions. The negative-time branch of these solution constitutes the correct starting point for moving-brane pre-big-bang cosmology which may terminate in a brane collision. Whether or not this collision will turn pre-big-bang contraction of the universe into expansion, thereby generating a "graceful exit", must currently be viewed as an open problem.

The five-dimensional origin of these four-dimensional moving brane solution is not known at present except for a number of special cases. There are two four-dimensional solutions with $\epsilon=$ const which can be directly lifted to five dimensions [9]. A less trivial example of a five-dimensional moving brane solution which reduces to one of the above $D=4$ solutions in a specific limit only has been found more recently [10].

\section{Modelling three-branes with kinks}

Studying more complicated dynamics of three-branes, such as brane-brane or brane-boundary collision, requires a more microscopic understanding of $\mathrm{M}$ theory which is currently out of reach. However, these processes can be studied in the context of a toy model where the three-branes are replaced by smooth kink solutions of a bulk scalar field theory. The following action [11] for this toy model, replacing the M-theory action (1),

$$
\tilde{S}_{5}=-\frac{1}{2 \kappa_{5}^{2}}\left\{\int_{M_{5}} \sqrt{-g}\left[\frac{1}{2} R+\frac{1}{4} \partial_{\alpha} \Phi \partial^{\alpha} \Phi+\frac{1}{2} e^{-\Phi} \partial_{\alpha} \chi \partial^{\alpha} \chi+V(\Phi, \chi)\right]\right.
$$




$$
\left.+\int_{M_{4}^{1}} \sqrt{-g} 2 W-\int_{M_{4}^{2}} \sqrt{-g} 2 W\right\} .
$$

has been proposed recently. Here, the potential $V$ is obtained from a "superpotential" $W=e^{-\Phi} w(\chi)$ by

$$
V=\frac{1}{3} e^{-2 \Phi} w^{2}+\frac{1}{2} e^{-\Phi} U, \quad U=\left(\frac{d w}{d \chi}\right)^{2} .
$$

The potential $U$ for the new bulk field $\chi$ is required to be periodic with period $v$, that is $U(\chi+v)=U(\chi)$ and to have minima for all $\chi=n v$, where $n \in \mathbf{Z}$, with $U(n v)=0$. These conditions can be easily translated into conditions on the function $w(\chi)=\int_{0}^{\chi} d \tilde{\chi} \sqrt{U(\tilde{\chi})}$ which specifies the superpotential. An example for $U$ is provided by the sine-Gordon potential

$$
U=m^{2}\left[1-\cos \left(\frac{2 \pi \chi}{v}\right)\right],
$$

although the specific form of the potential is largely irrelevant for the subsequent discussion as long as the above general properties are satisfied.

How does (7) relate to the original M-theory action (1)? Consider first a situation where the field $\chi$ is constant throughout space-time and is located in the $n^{\text {th }}$ minimum of $U$, that is, $\chi=n v$. Eq. (7) then reduces to the M-theory action (1) without a three-brane, corresponding to a charge configuration

$$
\left(\beta_{1}, \beta_{2}, \beta_{3}\right)=(n,-n, 0),
$$

if the identification $\sigma=w(v)$ is being used.

The equations of motion derived from the action (7) have Bogomol'nyi-type first integrals. These first order equations can be used to obtain a BPS singlekink solution which interpolates between $\chi=n v$ on the first boundary and $\chi=(n+1) v$ on the second boundary. In a thin-kink approximation the $3+1-$ dimensional surface defined by the core of the kink can be described by a NambuGoto action and can, therefore, be identified with the three-brane in Eq. (1). Hence, in the background of a single kink, the action (7) reduces to the M-theory action with a single-charged three-brane, corresponding to charges

$$
\left(\beta_{1}, \beta_{2}, \beta_{3}\right)=(n,-(n+1), 1) .
$$

Note that different configurations of the scalar field $\chi$ in the defect model correspond to topologically distinct M-theory models. It has been shown [11] that a collision of a kink with a boundary generically leads to a transition between a vacuum state (11) with a single-charged three-brane and a vacuum state (10) without a three-brane. Topological transitions on the M-theory side can, therefore, be modelled by simple scalar field dynamics using the action (7). This opens up the possibility of modelling the evolution of more complicated configurations such as networks of three-branes in the early universe. 


\section{Acknowledgements}

The author is supported by a PPARC Advanced Fellowship.

\section{References}

[1] P. Horava and E. Witten, "Eleven-Dimensional Supergravity on a Manifold with Boundary," Nucl. Phys. B 475 (1996) 94 [hep-th/9603142].

[2] A. Lukas, B. A. Ovrut, K. S. Stelle and D. Waldram, "The universe as a domain wall," Phys. Rev. D 59 (1999) 086001 [hep-th/9803235].

[3] M. Brandle and A. Lukas, "Five-branes in heterotic brane-world theories," Phys. Rev. D 65 (2002) 064024 [hep-th/0109173].

[4] R. Donagi, A. Lukas, B. A. Ovrut and D. Waldram, "Non-perturbative vacua and particle physics in M-theory," JHEP 9905 (1999) 018 [hep-th/9811168].

[5] R. Donagi, B. A. Ovrut, T. Pantev and D. Waldram, JHEP 0108 (2001) 053 [hep-th/0008008].

[6] J. Derendinger and R. Sauser, "A five-brane modulus in the effective $\mathrm{N}=1$ supergravity of M-theory," Nucl. Phys. B 598 (2001) 87 [hep-th/0009054].

[7] G. W. Moore, G. Peradze and N. Saulina, instantons," Nucl. Phys. B 607 (2001) 117 [hep-th/0012104].

[8] E. Lima, B. A. Ovrut and J. Park, Nucl. Phys. B 626 (2002) 113 [hepth/0102046].

[9] E. J. Copeland, J. Gray and A. Lukas, "Moving five-branes in low-energy heterotic M-theory," Phys. Rev. D 64 (2001) 126003 [hep-th/0106285].

[10] E. J. Copeland, J. Gray, A. Lukas and D. Skinner, [hep-th/0207281].

[11] N. D. Antunes, E. J. Copeland, M. Hindmarsh and A. Lukas, [hepth/0208219]. 